\title{
Midline Thalamic Region: Widespread Excitatory Input to the Entorhinal Cortex and Amygdala
}

\author{
D. X. Zhang and E. H. Bertram \\ Department of Neurology, University of Virginia Health Science Center, Charlottesville, Virginia 22908
}

The midline thalamus has a role in memory formation and has well described projections to multiple limbic sites including the hippocampus, amygdala, and entorhinal cortex. Stimulation of this region evokes excitatory responses in the CA1 region of the hippocampus, but nothing is known about the nature of thalamic influence on other limbic sites such as the entorhinal cortex and the amygdala. In this study we electrically stimulated the midline thalamus in anesthetized rats to determine whether responses could be evoked in the amygdala or entorhinal cortex. In addition we examined the distribution of the responses within the target regions as well as the effect of short interval paired or high-frequency tetanizing stimulation. We found reproducible responses in the entorhinal cortex and the amygdala with a distribution of responses that matched the

The midline thalamus, including the medial dorsal nucleus, may play a significant role in certain types memory formation (Parker et al., 1997; Aggleton and Brown, 1999), and may be an important circuit component in limbic seizures (Patel et al., 1988; Miller and Ferrendelli, 1990; Hirayasu and Wada, 1992; Cassidy and Gale, 1998; Juhász et al., 1999; Bertram et al., 2001). The midline thalamic region has widespread connections throughout the brain (Herkenham, 1978; Yanagihara et al., 1987; Su and Bentivoglio, 1990; Wouterlood et al., 1990; Groenewegen and Berendse, 1994; Dolleman-Van der Weel and Witter, 1996). Many structures in the limbic system have well described pathways to the midline nuclei (Aggleton and Mishkin, 1984; Russchen et al., 1987; van Groen and Wyss, 1990; Kuroda et al., 1992; Reardon and Mitrofanis, 2000). These connections, which are often reciprocal, suggest that these thalamic nuclei could synchronize activity across limbic sites as the lateral thalamic nuclei synchronize cortical activity (Dempsey and Morison, 1942; Morison and Dempsey, 1942; Hunter and Jasper, 1949). However, there are few reports examining the effect these nuclei have on the limbic system (Dolleman-Van der Weel et al., 1997; Bertram and Zhang, 1999).

Early reports of thalamic influence on the cortex (Morison and Dempsey, 1942, 1943). described the recruiting response from repetitive stimulation of midline thalamus. There has been little work examining the effect of thalamic stimulation on limbic structures. Two studies of midline stimulation have shown a predominantly excitatory effect on CA1 neurons (Dolleman-Van der

Received Oct. 23, 2001; revised Jan. 29, 2002; accepted Feb. 7, 2002.

This study was supported by National Institutes of Health, National Institute of Neurological Disorders and Stroke Grant NS 25605. We thank John Williamson for his technical expertise.

Correspondence should be addressed to Edward H. Bertram, P. O. Box 800394, University of Virginia, Department of Neurology, Charlottesville, VA 22908-0394. E-mail: ehb2z@virginia.edu.

Copyright (ㄷ) 2002 Society for Neuroscience $\quad 0270-6474 / 02 / 223277-08 \$ 15.00 / 0$ described synaptic input from the thalamus. In addition, highfrequency stimulation induced a consistent long-term potentiation in the two sites. Paired stimulation resulted in depression of the test response in the amygdala, but a facilitation in the entorhinal cortex. These findings indicate that the midline has a significant monosynaptic excitatory influence in the amygdala and the entorhinal cortex. Combined with the previous work in the hippocampus, this study suggests that the midline thalamus plays a significant role in limbic physiology and may serve to synchronize activity in this system.

Key words: thalamus; amygdala; entorhinal cortex; physiology; long-term potentiation; thalamolimbic regional interactions; limbic system

Weel et al., 1997; Bertram and Zhang, 1999). In addition, the latter study suggested that the thalamic input had a slightly different effect on CA1 responses compared with CA3 induced responses. Long-term potentiation (LTP) in CA1 from stimulation in either CA3 or the thalamus was pathway-specific, an observation that has also been made in the lateral nucleus of the amygdala (McKernan and Shinnick-Gallagher, 1997; Weisskopf and LeDoux, 1999; Heinbockel and Pape, 2000). The importance of studying the different excitatory inputs to a region was emphasized by studies that revealed a different pharmacology for LTP in the amygdala depending on whether the thalamic (medial geniculate nucleus) or cortical inputs were stimulated (Weisskopf et al., 1999).

The reciprocal anatomic connections between the midline thalamic region and multiple limbic sites such as the hippocampus, entorhinal cortex, and the amygdala(Aggleton and Mishkin, 1984; Yanagihara et al., 1987; Su and Bentivoglio, 1990; Wouterlood et al., 1990; Turner and Herkenham, 1991; Kuroda et al., 1992; Reardon and Mitrofanis, 2000) suggest that this circuit may have important roles in emotion and memory. LTP work in the amygdala as well as our work examining thalamic influence over the hippocampus suggests that the thalamic excitatory connections are physiologically and pharmacologically unique. For these reasons it is important to determine how the midline thalamus influences the amygdala and entorhinal cortex. In this study we demonstrate that there are widespread monosynaptic excitatory responses in the amygdala and the entorhinal cortex after thalamic stimulation, and that some of these responses are regionally distinct.

\section{MATERIALS AND METHODS}

Animal preparation. The major methods are similar to our previous paper describing the hippocampal response to thalamic stimulation (Bertram and Zhang, 1999). In the present study, a total of 41 adult male Sprague 
Dawley rats (300-450 gm; Hilltop Laboratories, Scottsdale, PA) were used under a protocol approved by the Animal Research Committee of the University of Virginia following United States Department of Agriculture (USDA) and Helsinki guidelines. Twenty-one rats were used to study the thalamic projection to the entorhinal cortex, and the remaining 20 rats were used in the examination of the projection to the amygdala. The rats were anesthetized with urethane $(1.2 \mathrm{gm} / \mathrm{kg}$, i.p. $)$ and placed on a multi arm stereotaxic frame. The body temperature was maintained at $37^{\circ} \mathrm{C}$ by a water blanket. At the end of the experiments, the rats were killed by decapitation while still anesthetized, and the brains were removed to confirm electrode placement.

The electrodes were inserted into the brain stereotactically using target coordinates from a standard atlas (Paxinos and Watson, 1986). The bite bar for these experiments was set at $-3.3 \mathrm{~mm}$. A thalamic twisted pair bipolar stainless steel stimulating electrode insulated with a Teflon coating with only the cut ends exposed (wire diameter, $0.125 \mathrm{~mm}$; tip separation, $1.0 \mathrm{~mm}$ ) was placed in the midline thalamus $(1.8-2.3 \mathrm{~mm}$ posterior to bregma, lateral $0.4-0.8 \mathrm{~mm}$ to the midline, $5.5-6.3 \mathrm{~mm}$ below the dura, with a $5^{\circ}$ arm angle from vertical axis for the entorhinal cortex experiments and a $0^{\circ}$ angle for the amygdala experiments). This position was in the lower aspect of the medial dorsal nucleus or just above the rhomboid and reuniens nuclei. The recording electrodes were glass micropipettes filled with $0.9 \% \mathrm{NaCl}$ and $1 \%$ Fast Green. To help position the thalamic stimulating electrode, an additional recording electrode was placed into the CA1 pyramidal cell layer $(5.4-5.6 \mathrm{~mm}$ posterior to bregma, $4.8-5.0 \mathrm{~mm}$ lateral to the midline, and $2.3-2.5 \mathrm{~mm}$ below the dura) to record the maximal thalamic induced CA1 response after adjusting thalamic stimulating and CA1 recording electrode depths. The intensity of thalamic stimulation (duration, $0.2 \mathrm{msec}$; monophasic) inducing a maximal CA1 response was used as the initial stimulating intensity of the thalamic projection to entorhinal cortex and the amygdala.

Basic characteristics and topography of responses. The purpose of this series of experiments was to define the basic parameters of the responses in the entorhinal cortex and the amygdala at defined anteroposterior and mediolateral positions. The characteristics under examination included the presence of a response, the presence of a population spike, and the latencies to the peaks of significant early responses. These observations were compared with the responses obtained in CA1 after thalamic stimulation. In some animals the responses were recorded from a minimum of two anteroposterior planes separated by at least $0.5 \mathrm{~mm}$. In the entorhinal cortex the nominal anteroposterior placements were (relative to bregma) -6.3 (five rats), -6.8 (12 rats), -7.3 (five rats), and $-7.8 \mathrm{~mm}$ (two rats). Additional recordings were also made at -8.3 (four rats) and $-8.8 \mathrm{~mm}$ (two rats). The regular mediolateral coordinates for the recording electrodes were separately $6.3,6.0,5.5,5.0,4.5,4.0$, and $3.8 \mathrm{~mm}$ from midline to either side. The depth of the recording electrode was from 6.5 to $8.3 \mathrm{~mm}$ below the dura with the depth increments of $0.5 \mathrm{~mm}$, in some cases $0.3 \mathrm{~mm}$. However, for the more posterior regions the electrode depth was more limited by the changing configuration of the skull, which resulted in a shorter distance from dura to entorhinal cortex.

The nominal placements for the amygdala recording electrodes were -2.8 (19 rats) and $-3.3 \mathrm{~mm}$ (seven rats). The lateral electrode placement was $3.5,4.0,4.5$, and $5.0 \mathrm{~mm}$ from midline and $6.5-9.5 \mathrm{~mm}$ in $0.5 \mathrm{~mm}$ increments below the dura. In a subset of animals, responses were recorded in the same plane bilaterally to determine whether the responses were similar on both sides. All described procedures were not performed in all rats.

Each collected response is an average of five consecutive responses induced by stimuli delivered once every $10 \mathrm{sec}$. Responses were evaluated, as noted above, for latency and maximal amplitude, as well as for the presence of a population spike. In each anteroposterior plane, the relative position of the response with the shortest latencies and maximal amplitudes (as determined by the maximal amplitude negative responses) was noted.

Long-term potentiation. LTP is generally considered to be a monosynaptic process that is induced by several brief, high-frequency stimuli (Schwartzkroin and Wester, 1975; Buzsáki and Eidelberg, 1982; Bliss et al., 1983). We had previously shown that the thalamic input to CA1 can induce this enhanced response in CA1 (Bertram and Zhang, 1999). In this study LTP was used to determine whether the thalamic projection to the entorhinal cortex or amygdala had the potential to induce this monosynaptic response. This issue is important as it is possible that some of the responses, especially those with long latencies, may be polysynaptic.

The conditioning stimulus consisted of two trains (separated by $10 \mathrm{sec}$ ) of 100 pulses delivered at $100 \mathrm{~Hz}$. The intensity for the conditioning stimulus was the same as for the baseline and test stimulus single pulses. The intensity for the single stimuli were set to provide a response that was approximately half of the maximal amplitude so that there was potential for a potentiation of the response. The responses were evaluated by amplitude and peak latency of the largest negative response (Fig. $1, N 2$ ), and after the conditioning trains, these characteristics were compared with the baseline responses as percentage of change.

Paired-pulse studies. Paired stimuli given at short intervals (e.g., 20 $\mathrm{msec}$ ) in the hippocampus are a synaptic response that results in a second (or test) response that is typically smaller than the first (an observation usually called paired-pulse inhibition or depression). We had previously found that thalamic stimulation of sufficient intensity could induce paired-pulse depression in the CA1 region of the hippocampus, although this depression was less than usually observed after contralateral CA3 stimulation (Bertram and Zhang, 1999). In this study we wished to determine whether paired thalamic stimulation had a similar effect in the entorhinal cortex and amygdala.

Stimulation at $20 \mathrm{msec}$ interval was used to determine whether there was any difference in the responses to thalamic stimulation between CA1 pyramidal cell layer and entorhinal cortex or amygdala. Before the recording electrode was placed into entorhinal cortex or amygdala, the CA1 responses to paired-pulse thalamic stimulation were recorded. After the recording electrode was placed into entorhinal cortex or amygdala, the responses to paired-pulse thalamic stimulation were recorded again. These responses were obtained in the region of each AP plane that provided the maximal response after thalamic stimulation. The intensities of test and conditioning stimulation were set at levels sufficient to induce a maximum conditioning response, because we have found that this intensity provides the most consistent and reliable results. Responses were evaluated for change in amplitude of the primary negative potential (Fig. 1A, N2). We chose a standardized AP plane for this analysis for the entorhinal cortex (EC) (bregma $-6.8 \mathrm{~mm})(n=12)$ and the amygdala (bregma $-2.8 \mathrm{~mm})(n=3)$. For purposes of comparison of change in the test response among CA1, EC, and amygdala, a percentage of change [(test response amplitude - conditioning response amplitude)/conditioning response amplitude $\times 100 \%$ ] for each paired stimulation was calculated. The means of these percent changes were calculated, and EC and amygdala were each separately compared with the percentage of change in CA1 using a $t$ test. The results are reported in Table 3, under the percentage of change column.

Histology. At the end of each experiment, the electrode positions were marked for histological confirmation. The Fast Green in the recording electrode was iontophoresed into the surrounding tissue by using negative direct current (20-50 $\mu \mathrm{A} ; 5-10 \mathrm{~min})$ at the end of the experiment. The positions of the recording electrode in that AP plane were extrapolated from this one position. Positive direct current ( $10 \mathrm{~V}$ for 5-10 sec) was passed through the negative tip (lower) of the stimulating electrodes to deposit iron from the electrode into the surrounding tissue. The animals were decapitated while still under anesthesia, and the brains were removed and placed into fixative consisting of $1 \%$ potassium ferrocyanide and $4 \%$ formaldehyde. The fixed brains were frozen and sectioned at $40 \mu \mathrm{m}$. The sections were stained for Nissl with thionine. The electrode positions with the maximal response were determined by the position of the iontophoresed Fast Green, and the other electrode positions more were determined by extrapolation based on the distance moved between recording positions. Each position was plotted on a standard diagram for each AP plane of study, and the responses recorded were correlated to each position (Paxinos and Watson, 1997).

Statistical evaluation. All data are presented as means \pm SEM. Quantitative comparisons were made in the paired-pulse and long-term potentiation experiments. For the paired-pulse data, the percentage of change in amplitude of the second or test response was compared between either the amygdala or entorhinal cortex and the hippocampal CA1 response. The percentage of change was determined for each individual response, and the two groups (either CA1 and amygdala or CA1 and entrorhinal cortex) were compared with a $t$ test.

For the LTP experiments data after the conditioning stimulation (percentage of change in amplitude or latency) were compared with the normalized values obtained immediately before conditioning with a one-way ANOVA with repeated measures (ANOVA-RM). Post hoc pairwise comparisons between the last baseline value and each of the postconditioning points were made with a Student-Newman-Keuls test. For all comparisons significance was set at a level of $p<0.05$. 

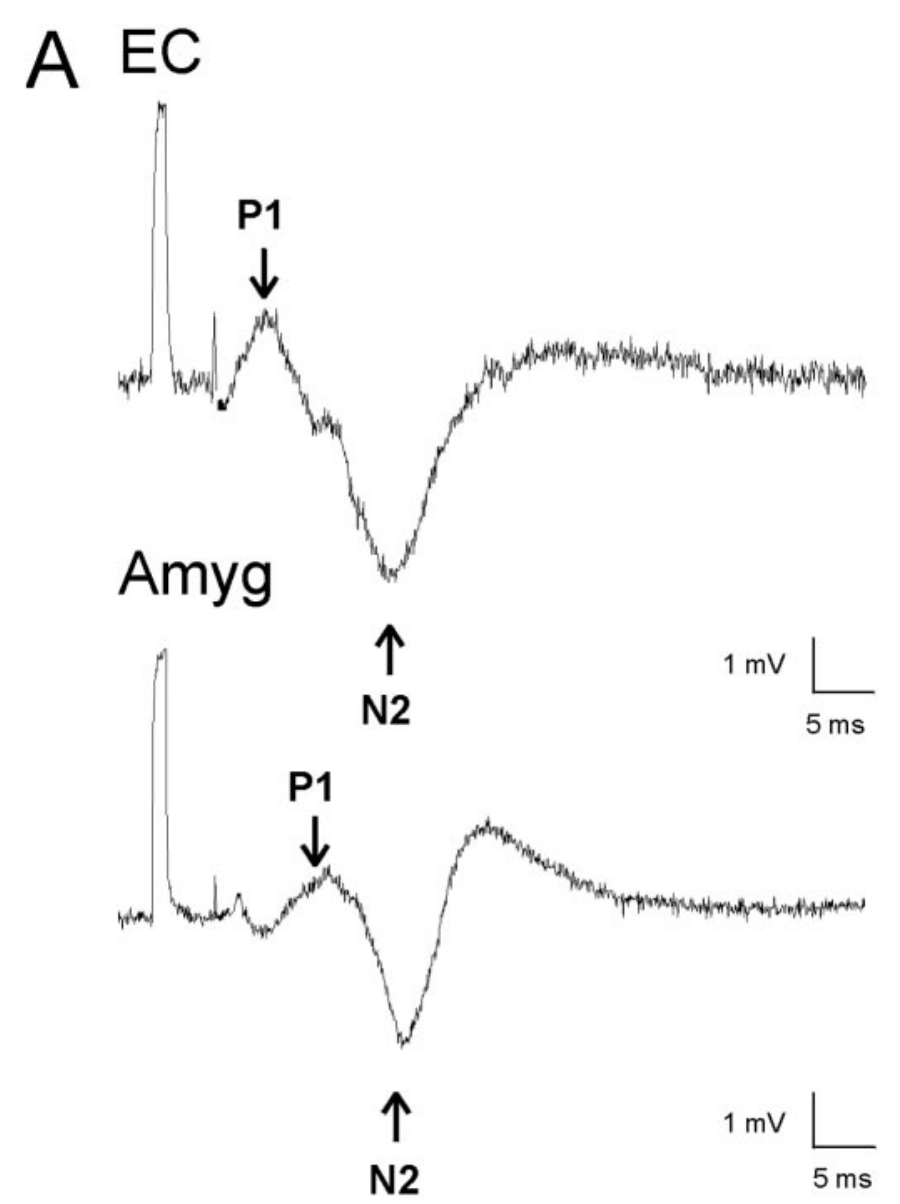

B
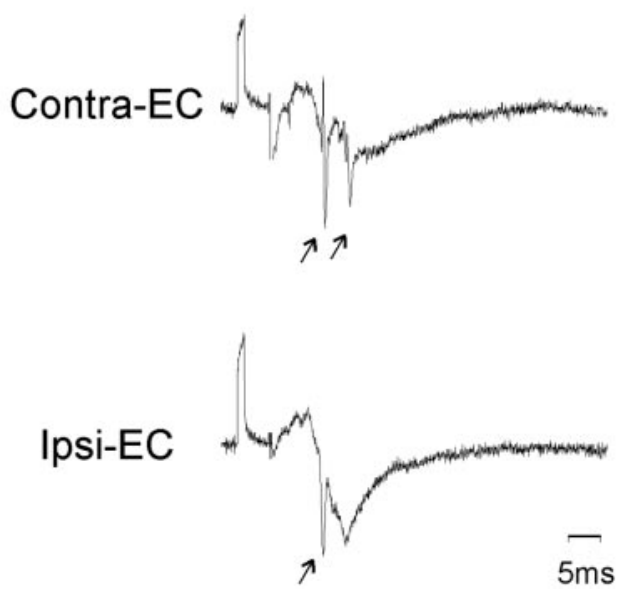

Figure 1. Basic field responses. A, Typical EC and amygdala responses to midline thalamic stimulation. Most early responses were an initial positive deflection $(P 1)$ followed by a larger negative wave (N2). After the stimulus there was a variable initial negative potential that was not included in the evaluation. $B$, Bilateral response in EC with superimposed population spike after stimulation at a single point in the midline thalamus. For some of the evoked responses, there was a well developed spike discharge mixed in with or superimposed on the N2 wave (arrow). This latter response was not seen consistently in all animals. When recorded bilaterally, similar responses were found ipsilateral and contralateral to stimulation. In each trace there is an initial positive $5 \mathrm{mV}$ calibration pulse followed several milliseconds later by a lower amplitude stimulation artifact that is partially blanked.

\section{RESULTS}

\section{Basic response characteristics}

Responses were obtained throughout the EC and the amygdala. Although there was some variation in the qualitative features of the field response, the overall morphology of the maximal amplitude responses in the amygdala and EC was quite similar. For some responses there was an initial low-amplitude negative response (N1) that was quite variable in its presence and appearance, but was found in the amygdala and EC without predilection for a particular region or site within a region. The first consistent response across animals and sites was a positive response (P1) (Fig. 1A). This response was followed by a higher amplitude negative wave (N2). The responses increased in amplitude with increasing stimulus intensity (Fig. $2 A, B$ ). On occasion, negative spikes or multiple spikes were superimposed on N2 (for examples, see Figs. $1 B, 2 A$ ), and in general these spikes were only seen when $\mathrm{N} 2$ was at or near maximal amplitude (Fig. $2 A$ ).

In comparing the latency and amplitudes of the EC and amygdala responses to the CA1 response in the hippocampus the latency to onset of the response was similar, but the peak latencies for N2 are longer. The N2 response was also longer in duration and lower in amplitude in EC and amygdala compared with CA1 (see Table 1 for latency and amplitude data).

The responses were seen throughout the EC and amygdala, but there was usually an area of maximal amplitude and shortest latency in each AP plane examined. For the EC the responses tended to be higher in amplitude in the more lateral areas of layers II and III. There was a clear phase reversal as the electrode moved into the superficial portions of layer II and layer I. (Figs. $2 A, 3)$. For the amygdala the responses were of highest amplitude and shortest latency in the area around the basal nucleus (lateral mid to ventral aspect of the amygdala) (Figs. 2B, 3). The mediolateral differences in response were less pronounced in EC than in amygdala (Fig. 3). As noted above there was a clearly graded response to increasing stimulus intensity, with a likely postsynaptic response that evolves to a higher amplitude response with occasional superimposed spike discharges (Fig. 2A,B). As seen in Table 2 the responses were seen with similar latencies and amplitudes throughout the anteroposterior extent of the EC and amygdala.

Within the midline thalamus there was a region that clearly elicited the maximal responses in EC and amygdala when stimulated. As shown in Figure 4 this region corresponded to the ventral aspects of the MD, with some extension into the upper rhomboid nucleus. This thalamic region was similar to the midline thalamic region that elicited the maximal response in CA1. When the stimulating electrode was placed $2 \mathrm{~mm}$ laterally from the midline and moved ventrally through the lateral dorsal and ventrolateral nuclei, we could not evoke responses in EC and amygdala (data not shown).

In a small number of animals we recorded in the EC and amygdala on both sides (ipsilateral and contralateral to stimulation). We found responses that were essentially identical on the two sides. See Figure 1B for responses in the EC ipsilateral and contralateral to stimulation.

\section{Paired-pulse studies}

Short-interval paired stimulation evoked opposite responses in the EC and the amygdala. The amygdala responded like CA1 with a significant suppression of $\mathrm{N} 2(p<0.05 ; t$ test $)$. On the other hand, EC had a significant facilitation of the response $(p<0.001$; $t$ test). (Fig. 5, Table 3). These responses were obtained at a 

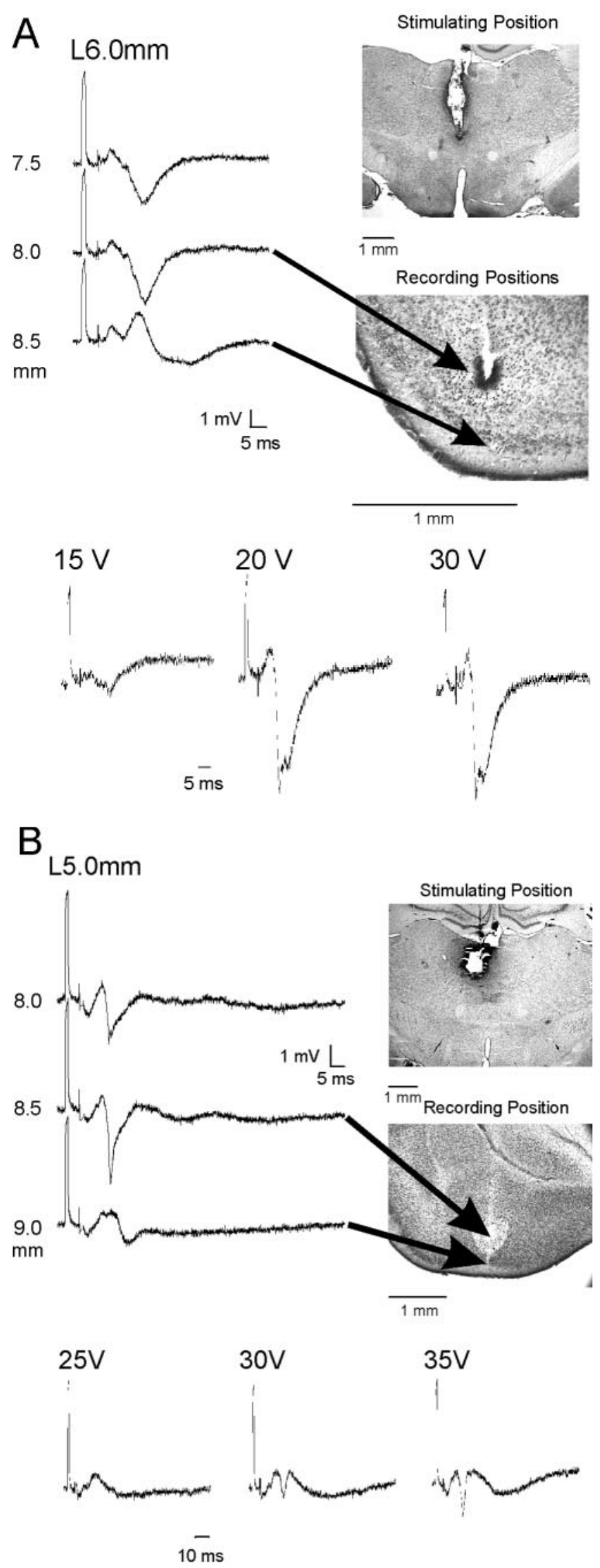

Figure 2. Basic depth profile of EC and amygdala response. A, EC responses recorded at $-6.8 \mathrm{~mm}$ posterior to the bregma, $6.0 \mathrm{~mm}$ lateral
Table 1. Latencies and amplitudes of primary responses in CA1, EC and amygdala

\begin{tabular}{|c|c|c|c|}
\hline \multirow[b]{2}{*}{ Recording site $(n)$} & \multicolumn{2}{|c|}{ Latency (msec) } & \multirow{2}{*}{$\frac{\begin{array}{l}\text { Amplitude } \\
(\mathrm{mV})\end{array}}{\mathrm{N} 2}$} \\
\hline & $\mathrm{P} 1$ & $\mathrm{~N} 2$ & \\
\hline CA1 (20) & $9.76 \pm 0.29$ & $6.71 \pm 0.33$ & $16.10 \pm 1.09$ \\
\hline $\mathrm{EC}(12)$ & $4.47 \pm 0.24$ & $13.12 \pm 0.44$ & $3.38 \pm 0.27$ \\
\hline Amyg (9) & $8.88 \pm 0.49$ & $15.65 \pm 1.16$ & $2.81 \pm 0.44$ \\
\hline
\end{tabular}

Values are means \pm SEM.

Shortest latency, highest amplitude response in each animal.

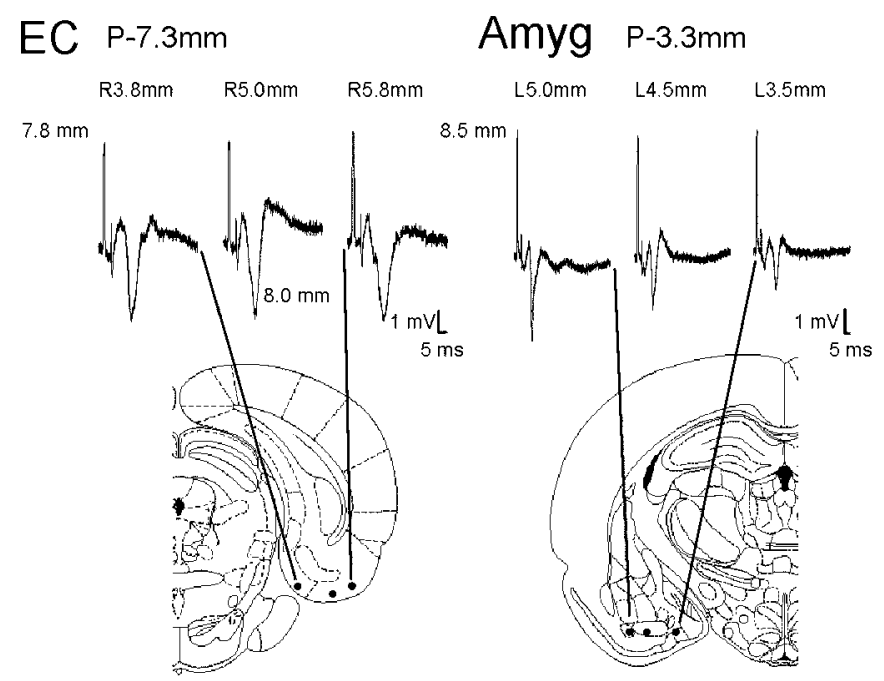

Figure 3. Mediolateral distribution of EC and amygdala responses. Responses to thalamic stimulation recorded $\sim 0.5 \mathrm{~mm}$ posterior to responses shown in Figure 2. Responses are obtained at depth that gives approximately the greatest amplitude response. There is little shift in EC amplitude in the three mediolateral positions, but for the amygdala the greatest amplitude is consistently in the more lateral positions. Brain diagrams from Paxinos and Watson (1997).

maximal conditioning response and were the same throughout the EC and amygdala. The data displayed are from a standardized site in one AP plane for the EC and amygdala, but the relative nature of the paired response was similar throughout each region.

\section{Long-term potentiation}

LTP was induced in the EC and amygdala (Fig. 6). For the amygdala $(n=5)$ the baseline amplitude for the $\mathrm{N} 2$ response was $2.95 \pm 0.69 \mathrm{mV}$, and the latency was $18.22 \pm 0.12 \mathrm{msec}$. There was a significant increase in the amplitude $(p<0.001 ; F=11.295$; between group $\mathrm{df}=7$ ) and a significant decrease in the latency

$\leftarrow$

from midline. Maximal evoked responses are shown at each of the depths, and the electrode tract and position are shown in the adjacent micrograph. Note that the polarity changes at the deepest position. Arrows point to the approximate position along electrode tract from which responses were recorded. Darker area around tract at the top arrow is Fast Green to indicate site of recording. In the bottom part of figure the three traces obtained from one depth in another animal show the graded response to increasing stimulation intensity. $B$, Amygdala responses recorded -2.8 $\mathrm{mm}$ posterior to bregma, $5.0 \mathrm{~mm}$ lateral from midline. Electrode depths and positions as for EC in $A$. Graded response to increasing stimulation intensity shown at bottom of figure. 
Table 2. Amplitudes and latencies from different anterior posterior planes

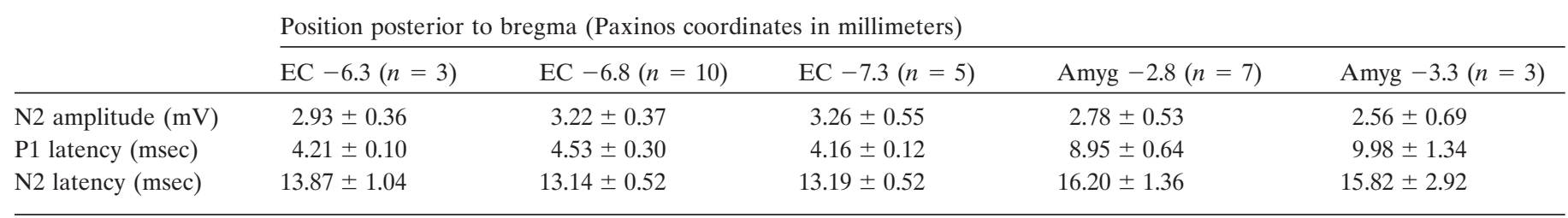

Values are means \pm SEM. Some animals provided data at more than one AP position.

Shortest latency, greatest amplitude from each plane.

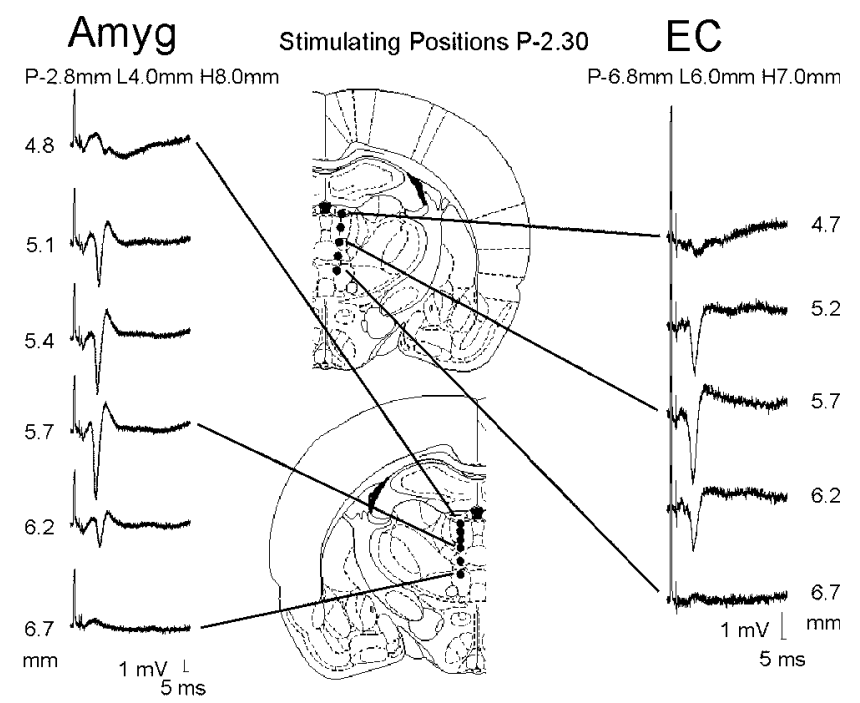

Figure 4. EC and amygdala responses to different midline thalamic stimulation positions. The numbers next to the responses are the approximate positions of the negative (bottom) tip of the stimulating electrode. The extent of the stimulating electrode trajectory was confirmed histologically. The recording electrodes were kept at the indicated positions as the stimulating electrode was moved through the thalamus. These observations indicate that the midline site for eliciting responses in the $\mathrm{EC}(n=$ 4 ) and amygdala $(n=3)$ is restricted to a narrow area. Brain diagrams from Paxinos and Watson (1997).

$(p<0.001 ; F=36.772$; between group df $=7)$. Post hoc pairwise comparison (Student-Newman-Keuls) between baseline and each of the points after conditioning reveal that each amplitude and latency measure after conditioning was significantly different from baseline ( $p<0.001$ for all points, amplitude and latency).

For the EC $(n=7)$ the baseline amplitude was $2.30 \pm 0.28 \mathrm{mV}$, and the latency was $13.00 \pm 0.72 \mathrm{msec}$. There was also a significant increase in amplitude of the response after conditioning $(p=0.004, F=3.686$, between group $\mathrm{df}=7)$ and a significant decrease in latency $(p=0.01, F=3.166$, between group $\mathrm{df}=7)$. Post hoc Student-Newman-Keuls testing showed that all postconditioning latency changes were significantly less than baseline ( $p<0.04$ for all points). For amplitude, the post hoc pairwise comparison indicated that the first three points and the last measured point after conditioning were significantly greater than baseline ( $p<0.04$ for these four points).

\section{DISCUSSION}

The major finding of this study is the excitatory response in the amygdala and the entorhinal cortex that follow midline thalamic stimulation. This finding, together with our previous description of similar excitatory responses in the CA1 region of the hippocampus, suggests that the midline thalamic nuclei can play a
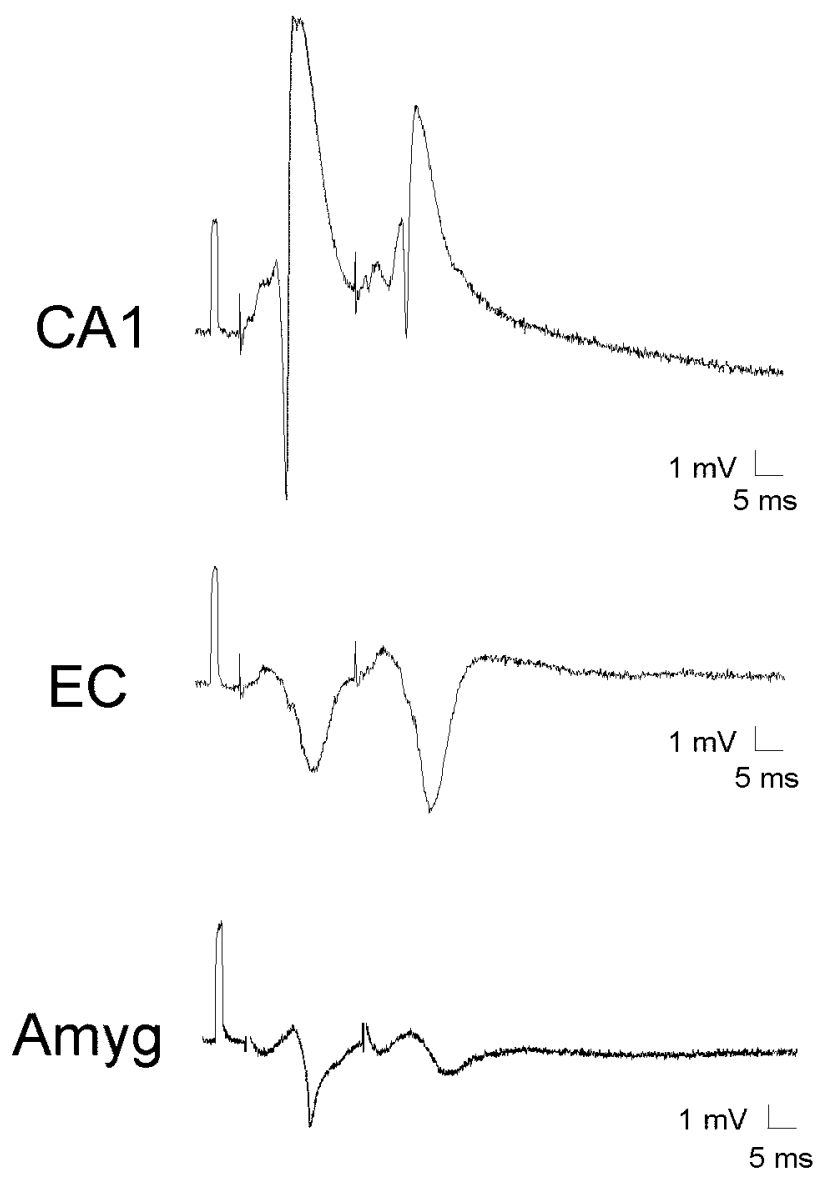

Figure 5. Short-interval $(20 \mathrm{msec})$ paired-pulse stimulation. Stimulus intensities were adjusted to achieve maximal response by midline thalamic stimulation. The evoked CA1 responses show the well described depression of the test response to thalamic stimulation. In comparison, the test EC response by the same thalamic stimulation shows a significant facilitation. Like the CA1 response, the amygdala shows a significant suppression of the test response. The data for these responses are shown in Table 3.

significant role in the physiology of these major limbic regions. The circuitry within and between each region is complex. The interactions between several regions (e.g., the hippocampus and entorhinal cortex) are well described, and these observations raise the possibility that the recorded responses are polysynaptic (e.g., a thalamically induced CA1 response that subsequently evokes an EC response). However, the LTP studies, which demonstrated potentiation in the EC and amygdala, suggest that the primary responses that we recorded were monosynaptic (Schwartzkroin and Wester, 1975; Buzsáki and Eidelberg, 1982; Bliss et al., 1983). The paired-pulse studies, with inhibition in CA1 and amygdala and potentiation in EC, suggest that there are regional variations 
Table 3. Short-interval paired-pulse response amplitude changes

\begin{tabular}{|c|c|c|c|c|}
\hline \multirow[b]{2}{*}{ Stimulation site $(n)$} & \multirow[b]{2}{*}{ Conditioning } & \multicolumn{2}{|c|}{ Amplitude (mV) } & \multirow[b]{2}{*}{$\%$ Change } \\
\hline & & Test & Absolute change & \\
\hline CA1 (17) & $15.98 \pm 1.19$ & $10.83 \pm 1.53$ & $-5.15 \pm 1.14$ & $-33.4 \pm 6.9$ \\
\hline $\mathrm{EC}(12)$ & $3.09 \pm 0.30$ & $3.90 \pm 0.40$ & $+0.81 \pm 0.21$ & $+27.3 \pm 7.1^{* * *}$ \\
\hline Amyg (3) & $2.18 \pm 0.98$ & $0.76 \pm 0.42$ & $-1.41 \pm 0.57$ & $-73.8 \pm 13.2^{*}$ \\
\hline
\end{tabular}

Values are means \pm SEM. Comparing percentage of change in either amygdala or EC to CA1.

${ }^{*} p<0.05 ; * * * p<0.001$.

in the evoked response that may influence the nature of the thalamic modulation of these different limbic areas.

The efferents from the midline thalamus to the limbic system are widespread, but within each region there are areas that receive a greater proportion of terminals from the thalamus, as assessed by tract tracing studies. In the hippocampus, the majority of the thalamic afferents are found in apical dendrites of CA1, as well as in the subiculum (with the subiculum sending axons back to the medial dorsal thalamic nucleus) (Herkenham, 1978; Wouterlood et al., 1990). In amygdala the majority of the midline thalamic afferents are in the central and basal nuclei (Su and Bentivoglio, 1990; Turner and Herkenham, 1991), and in EC the axons are more diffusely distributed throughout the structure, although there is some preference for the lateral as opposed to the medial EC and for the middle layers of the cortex (II, III, and IV) (Yanagihara et al., 1987; Wouterlood et al., 1990). This described anatomic distribution corresponds loosely to the areas of maximal response and phase reversal in the amygdala and EC. In the amygdala, maximal response was consistently found in the lateral regions in or near the basal nucleus, with a fall off in amplitude as the electrode moved medially (Fig. 3). In addition, there was a clear phase reversal as the recording electrode was moved ventrally below the basal nucleus (Fig. $2 B$ ), an observation suggesting that the fields were generated within that region of the amygdala, although it is possible that neighboring nuclei could contribute to the findings. In the EC there was less medial lateral difference (Fig. 3) but the maximal amplitude was found near layer III, with a phase reversal of the response as the recording electrode moved toward layer II (Fig. 2A). Although these findings support the concept that the responses are locally generated, they do not indicate directly whether the responses are monosynaptic or whether other regions may be contributing to these responses. However, the thalamic input to these adjacent areas (such as the piriform cortex) are much less well developed and defined. In the future a more precise analysis that includes the mapping of voltage fields in smaller increments and the recording of single unit responses will help resolve some of these issues.

The projection neurons within the midline, on the basis of retrograde tract tracing studies, have predominantly unilateral projections with only minimal crossing of midline ( $\mathrm{Su}$ and Bentivoglio, 1990; Dolleman-Van der Weel and Witter, 1996). There is some segregation of the midline neurons with regard to their target limbic regions with the more ventral nuclei (reuniens and rhomboid) projecting to the hippocampus and entorhinal cortex and the more dorsal nuclei (paraventricular and medial dorsal) projecting to the amygdala. However, there is evidence that there is overlap along the dorsoventral axis of the midline, because there are ventral neurons with projections to the amygdala and dorsal neurons with connections to the entorhinal cortex and hippocampus (Yanagihara et al., 1987; Su and Bentivoglio, 1990).
There is some evidence in the cat that there are other sites within the thalamus that may project to the hippocampus and entorhinal cortex (Yanagihara et al., 1987), but the majority of thalamolimbic projections originate in or near the midline.

It is of some note that the stimulus intensity to elicit maximal responses was somewhat higher than is seen for evoking responses in the hippocampus when stimulating locally, as we have seen in previous studies (Bertram and Zhang, 1999). This observation may be the result of the more widespread distribution of the projection neurons, so that it requires a more intense stimulus to recruit a sufficient number of neurons to allow a field potential to be recorded. We believe this was a reason why we found a much greater response in CA1 after thalamic stimulation compared with the response recorded by Dolleman-Van der Weel et al. (1997): we used a slightly greater intensity with an electrode type that stimulated a somewhat greater tissue volume (Bertram and Zhang, 1999). The lower amplitude response that we recorded in the amygdala and entorhinal cortex compared with CA1 was likely the result of a more dispersed cell population in the first two regions compared with the dense neuronal packing in CA1. The fact that we could record responses bilaterally from a single stimulation site, although there is strong anatomic evidence for ipsilateral segregation of projections, is probably a consequence of the relative nearness of the contralateral midline neurons to the stimulating electrode: it is not possible to deliver a stimulus sufficient to induce an ipsilateral response without stimulating the opposite side.

The relatively high stimulus intensities necessary to evoke responses in the target site may raise concerns that the responses were the result of the inadvertent stimulation of a site adjacent to the midline thalamic region or of fibers that traversed the stimulated region. These concerns may be partially ameliorated by the observations that were made in Figure 4: there was a narrow window in the midline region for eliciting these responses. Although we show data for midline electrode positions, we also found that electrodes placed $2 \mathrm{~mm}$ lateral to the midline failed to elicit responses. We cannot completely exclude the possibility that some fibers of passage might be inadvertently stimulated, but there are no such fibers, to our knowledge, in the region of the thalamus that elicits maximal response. Even if there were, the observation that stimulation in a single central site evokes excitatory responses from the three separate limbic sites is unique and emphasizes the potential role this region has in limbic system function.

There may also be concerns about the regions in the EC and amygdala that generate the responses, that what we are recording are far field responses generated by neighboring structures. It is difficult to determine a well defined point of maximal response. That difficulty may be related, in part, to the rather diffuse and widespread pattern of thalamic axon terminals seen throughout 


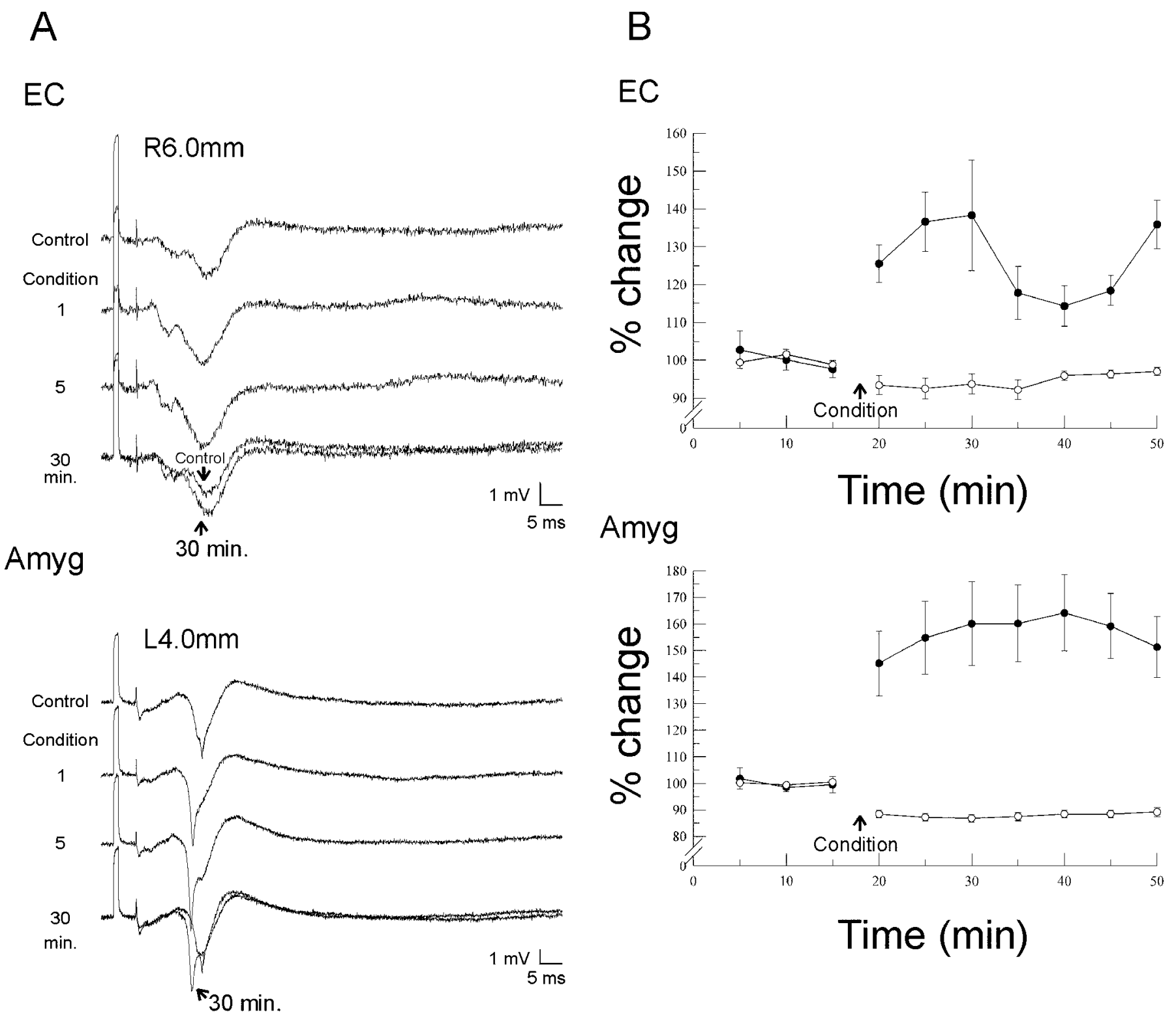

Figure 6. Long-term potentiation. A, Long-term potentiation in the EC and amygdala. After the conditioning stimuli, there is a significant and sustained (at least 30 min after conditioning) increase in amplitude of the EC and amygdala response ( filled circles). There is also a significant shortening of the latency in the amygdalar response, whereas there is only a slight decrease in latency in the EC (open circles). B, Means of amplitudes and latencies for EC $(n=7)$ and amygdala $(n=5)$, reflecting the changes after the conditioning stimuli. Bars indicate SEM. For the amygdala there was a significant shortening of the duration and an increase in the amplitude ( $p<0.001$ ANOVA-RM for the two measures). For the EC there was a significant increase in amplitude ( $p=0.004$ ANOVA-RM) and a significant decrease in peak latency $(p=0.01$ ANOVA-RM).

the EC and amygdala as well as the more widely distributed neuronal populations in these two regions, compared with the more tightly distributed pyramidal cells of CA1. The observation of reversals of polarity as the recording electrode was moved through the EC and amygdala (Figs. 2, 3). supports the hypothesis that the responses are generated locally in the two regions.

The primary observation of this study that there is a significant monosynaptic excitatory input from the thalamus to the entorhinal cortex and amygdala, combined with the previous studies of thalamic excitation of the hippocampus, suggests that the thalamus plays a significant role in the physiology of the limbic system. Because it has widespread connections to the limbic as well as cortical and subcortical structures, the midline thalamic region may have a powerful synchronizing role. Much work needs to be performed in follow up to this initial study with regard to the nature of these synapses, their location, and their pharmacology. There also needs to be an understanding of the thalamolimbic circuit, because, as with the thalamocortical circuit, there are major projections from these limbic sites back to the thalamus. Additional work examining the nature of the regional interactions within the thalamus is also necessary. An understanding of these pathways and circuits will no doubt give us a better understanding of the functions associated with these structures, including memory and limbic epilepsy.

\section{REFERENCES}

Aggleton JP, Brown MW (1999) Episodic memory, amnesia, and the hippocampal-anterior thalamic axis. Behav Brain Sci 22:425-444. 
Aggleton JP, Mishkin M (1984) Projections of the amygdala to the thalamus in the cynomolgus monkey. J Comp Neurol 222:56-68.

Bertram EH, Zhang DX (1999) Thalamic excitation of hippocampal CA1 neurons: a comparison with the effects of CA3 stimulation. Neuroscience 92:15-26.

Bertram EH, Williamson JM, Scott C, Mangan PS, Zhang DX (2001) The midline thalamus: alterations and a potential role in limbic epilepsy. Epilepsia 42:967-978.

Bliss TVP, Lancaster B, Wheal H V (1983) Long-term potentiation in commissural and Schaffer projections to hippocampal CA1 cells: an in vivo study in the rat. J Physiol (Lond) 341:617-626.

Buzsáki G, Eidelberg E (1982) Direct afferent excitation and long-term potentiation of hippocampal interneurons. J Neurophysiol 48:597-607.

Cassidy RM, Gale K (1998) Mediodorsal thalamus plays a critical role in the development of limbic motor seizures. J Neurosci 18:9002-9009.

Dempsey EW, Morison RS (1942) The production of rhythmically recurrent cortical potentials after localized thalamic stimulation. Am J Physiol 135:293-300.

Dolleman-Van der Weel MJ, Witter MP (1996) Projections from the nucleus reuniens thalami to the entorhinal cortex, hippocampal field CA1, and the subiculum in the rat arise from different populations of neurons. J Comp Neurol 364:637-650.

Dolleman-Van der Weel MJ, Lopes da Silva FH, Witter MP (1997) Nucleus reuniens thalami modulates activity in hippocampal field CA1 through excitatory and inhibitory mechanisms. J Neurosci 17:5640-5650.

Groenewegen HJ, Berendse HW (1994) The specificity of the "nonspecific" midline and intralaminar thalamic nuclei. Trends Neurosci 17:52-57.

Heinbockel T, Pape H-C (2000) Input-specific long-term depression in the lateral amygdala evoked by theta frequency stimulation. J Neurosci 20:RC68(1-5).

Herkenham M (1978) The connections of the nucleus reuniens thalami: evidence for a direct thalamo-hippocampal pathway in the rat. J Comp Neurol 177:589-610.

Hirayasu Y, Wada JA (1992) $N$-methyl-D-aspartate injection into the massa intermedia facilitates development of limbic kindling in rats. Epilepsia 33:965-970.

Hunter J, Jasper HH (1949) Effects of thalamic stimulation in unanaesthetised animals. EEG Clin Neurophysiol 1:305-324.

Juhász C, Nagy F, Watson C, da Silva EA, Muzik O, Chugani DC, Shah J, Chugani HT (1999) Glucose and [C-11]flumazenil positron emission tomography abnormalities thalamic nuclei in temporal lobe epilepsy Neurology 53:2037-2045.

Kuroda M, Murakami K, Kishi K, Price JL (1992) Distribution of the piriform cortical terminals to cells in the central segment of the mediodorsal thalamic nucleus of the rat. Brain Res 595:159-163.
McKernan MG, Shinnick-Gallagher P (1997) Fear conditioning induces a lasting potentiation of synaptic currents in vitro. Nature 390:607-611. Morison RS, Dempsey EW (1942) A study of thalamo-cortical relations. Am J Physiol 135:281-292.

Morison RS, Dempsey EW (1943) Mechanism of thalamocortical augmentation and repetition. Am J Physiol 138:297-308.

Parker A, Eacott MJ, Gaffan D (1997) The recognition memory deficit caused by mediodorsal thalamic lesion in non-human primates: a comparison with rhinal cortex lesion. Eur J Neurosci 9:2423-2431.

Patel S, Millan MH, Meldrum BS (1988) Decrease in excitatory transmission within the lateral habenula and the mediodorsal thalamus protects against limbic seizures in rats. Exp Neurol 101:63-74.

Paxinos G, Watson G (1986) The rat brain in stereotaxic coordinates, Ed 2. San Diego: Academic.

Paxinos G, Watson C (1997) Compact third edition: the rat brain in stereotaxic coordinates. San Diego: Academic.

Reardon F, Mitrofanis J (2000) Organisation of the amygdaladalothalamic pathways in rats. Anat Embryol 201:75-84.

Russchen FT, Amaral DG, Price JL (1987) The afferent input to the magnocellular division of the mediodorsal thalamic nucleus in the monkey, Macaca fascicularis. J Comp Neurol 256:175-210.

Schwartzkroin PA, Wester K (1975) Long-lasting facilitation of a synaptic potential following tetanization in the in vitro hippocampal slice. Brain Res 89:107-109.

Su H-S, Bentivoglio M (1990) Thalamic midline cell populations projecting to the nucleus accumbens, amygdala, and hippocampus in the rat. J Comp Neurol 297:582-593.

Turner BH, Herkenham M (1991) Thalamoamygdaladaloid projections in the rat: a test of the amygdala's role in sensory processing. J Comp Neurol 313:295-325.

Van Groen T, Wyss JM (1990) Extrinsic projections from area $\mathrm{CA}_{1}$ of the rat hippocampus: olfactory, cortical, subcortical, and bilateral hippocampal formation projections. J Comp Neurol 302:515-528.

Weisskopf MG, LeDoux JE (1999) Distinct populations of NMDA receptors at subcortical and cortical inputs to principal cells of the lateral amygdala. J Neurophysiol 81:930-934.

Weisskopf MG, Bauer EP, LeDoux JE (1999) L-type voltage-gated calcium channels mediate NMDA-independent associative long-term potentiation at thalamic input synapses to the amygdala. J Neurosci 19:10512-10519.

Wouterlood FG, Saldana E, Witter MP (1990) Projection from the nucleus reuniens thalami to the hippocampal region: light and electron microscopic tracing study in the rat with the anterograde tracer Phaseolus vulgaris-leucoagglutinin. J Comp Neurol 296:179-203.

Yanagihara M, Niimi K, Ono K (1987) Thalamic projections to the hippocampal and entorhinal areas in the cat. J Comp Neurol 266:122141. 